Rafael Villanueva Flores

\title{
Harina para bizcochos
}

\section{INTRODUCCIÓN}

A diferencia de los productos hechos con harina de trigos duros, los elaborados con harina de trigos suaves son generalmente menos densos, pero poseen una estructura interior más uniforme y mejores características de altura y esparcimiento. Las galletas, los wafers, los pretzels, los pasteles y los bizcochos son de calidad superior cuando son hechos con harina de trigos suaves y no utilizando harina de trigos duros.

Los términos duro y suave, cuando se aplican a los distintos tipos de trigo, se refieren a la textura del grano. Un grano de trigo duro requiere mayor fuerza para molerlo en comparación con uno de trigo suave. Durante la molienda, el endosperma de un trigo suave se fractura de manera diferente al de un trigo duro.

La harina de un trigo duro se caracteriza, a su vez, por poseer mayor cantidad de proteínas, mayor absorción de agua y granulometría más gruesa que la harina de un trigo suave. La harina de trigos duros que cuenta con un adecuado contenido de proteínas (1113\%, N X 5,7), como los de la especie Triticum aestivum, es principalmente utilizada para la producción de panes; la harina de los trigos de la especie Triticum durum son utilizados en la producción de pastas, y la harina con bajo contenido de proteínas (7-9\%), obtenida de la 
molienda de trigos suaves, de la especie Triticum aestivum, es adecuada para la producción de galletas y bizcochos.

Los trigos pueden clasificarse de varias maneras, pero el procedimiento más común es el que combina tres factores: a) la época del año en la cual son sembrados: los que se siembran en primavera, en invierno, hacia finales del verano o en otoño; b) el color de la superficie del grano: rojo (pigmentado) y blanco (sin pigmentación); c) la textura del endosperma: suave y duro. La mayoría de los trigos suaves son los de invierno, rojos o blancos.

\section{LOS BIZCOCHOS}

Los bizcochos pueden dividirse en tres tipos, dependiendo de la manera en la cual el aire se incorpora a la masa o batido. Mucha de la confusión encontrada en la literatura sobre el bizcocho es causada por no definir claramente el tipo de bizcocho al cual se está refiriendo.

El primer tipo de bizcocho es el que se hace mediante el denominado mezclado multietapa. Este es el procedimiento clásico, que empieza con la etapa del cremado. La grasa y el azúcar se mezclan para formar una crema, con el fin de incorporar aire en la grasa. Las dos o tres etapas de mezclado subsecuentes incorporan los líquidos y la harina para formar la masa o batido final. El cremado ofrece una serie de ventajas, como que pueden formarse un mayor número de células de aire, que lleva a que la textura sea más fina. El batido también puede fijarse en periodos más largos, pues el aire está en la grasa, donde permanece estable (inmóvil). Cuando la masa o batido del bizcocho es horneado y la grasa se disuelve, las células de aire son soltadas a la fase acuosa, donde los gases leudantes se difunden sobre ellas y consecuentemente leudan al bizcocho.

El segundo tipo de bizcocho es hecho con el mezclado de etapa única. Este tipo de bizcochos se adquiere en cajas; únicamente se le adicionan los líquidos y se mezcla. En este caso el aire es incorporado directamente en la fase acuosa, lo que se puede conseguir porque la mezcla contiene surfactantes que reducen la tensión superficial y permiten que el aire se incorpore directamente. El propilén glicol monoesterato es un surfactante común utilizado para este propósito. 
En el tercer tipo de bizcochos el aire es incorporado directamente en la fase acuosa mediante procedimientos mecánicos más que por la utilización de surfactantes. Es el tipo de bizcocho producido industrialmente, utilizando un batidor de alta velocidad.

Las propiedades de los bizcochos varían de acuerdo con el procedimiento de preparación utilizado. Por ejemplo, los bizcochos hechos por el procedimiento de cremado generalmente tienen un grano muy fino. Los bizcochos elaborados mediante el procedimiento de etapa única (de caja) son muy delicados y poco adecuados para transportarlos y comercializarlos una vez horneados, y en el caso de los bizcochos en los que el aire es incorporado directamente en la fase acuosa, el batido no es tan estable como el batido cremado.

El gas puede difundirse mediante el batido y, debido a que la presión en una burbuja pequeña es mayor que en una burbuja grande, hay la tendencia de las burbujas pequeñas a desaparecer y de las burbujas grandes a volverse más grandes (las burbujas grandes se comen a las pequeñas). Este fenómeno se controla por difusión: los gases se difunden desde las burbujas pequeñas hacia la fase acuosa y desde allí a las burbujas grandes. Debido a su mejor estabilidad, las burbujas grandes superan la alta viscosidad de la masa o batido, suben a la superficie y se pierden. Consecuentemente, estas masas o batidos de bizcochos no deben retenerse mucho tiempo antes del horneo.

Como alternativa a los bizcochos de caja (en los cuales el consumidor tiene que adicionar los líquidos, mezclar y hornear) existe la alternativa de los bizcochos prehorneados u horneados parcialmente. El proceso de horneado parcial consiste de tres etapas: fase de horneado, periodo de almacenamiento-despacho, y etapa de rehorneado u horneado final, llevado a cabo antes de su consumo. Durante la primera etapa los productos son horneados hasta que se forme una estructura que los haga capaces de resistir su almacenamiento y posterior distribución (son empacados a prueba de aire o bajo atmósfera controlada). La etapa de rehorneado revierte ciertas propiedades del producto desarrolladas durante el almacenamiento, generando características de un producto fresco, y para que sea conveniente para el consumidor, esta etapa no debe tomar mucho tiempo. Este tipo de productos requiere un mínimo de esfuerzo y de tiempo de preparación por parte del consumidor. Grau et al. (1999) reportaron la evaluación de un procedimiento de horneado en dos etapas para bizcochos esponjosos. 


\section{LA HARINA}

La harina para la elaboración de bizcochos es obtenida de la molienda de trigos suaves de invierno, rojos o blancos. La harina de trigo suave destinada a la producción de bizcochos usualmente contiene una combinación de aquellos pasajes de molienda con menor contenido de proteínas y cenizas. Tiene una extracción de $45 \%$ a $65 \%$ basada en la harina total. Esta harina, a pesar de tener granulometría promedio más fina que el resto de los pasajes, es reducida aún más mediante un molino de pines o por cualquier otro método y seguidamente tratada con gas cloro. Este tratamiento mejora sus características para la producción de bizcochos debido, en parte, a la despolimerización hidrolítica de la molécula de almidón, que resulta en una mayor capacidad de hidratación (Pyler, 1988).

La harina para bizcochos debe producir un gluten completamente suave durante el mezclado de la masa y totalmente exento de la dureza asociada con la masa para el pan. Por otro lado, el gluten debe poseer suficiente resistencia y ser adecuado en cantidad para garantizar la formación de una estructura de espuma fina en el bizcocho. Una harina típica para bizcochos tendrá el siguiente rango de especificación: proteínas: $8,5 \pm 0,5 \%$, cenizas: $0,36 \pm 0,04 \%, \mathrm{pH} 4,7 \pm 0,2$, tamaño de partícula: $10 \pm 0,5 \mathrm{~mm}$.

En la harina de trigos duros la absorción es gobernada por su contenido de proteínas y por la resistencia de la proteína, dos factores que ejercen un efecto mayor en el volumen de la hogaza del pan y en la calidad de la miga. La propiedad de absorción deseada en la harina para bizcochos es una elevada capacidad para acarrear líquidos. Esta propiedad, la cual de alguna manera es dependiente del contenido de proteínas de la harina, parece estar inicialmente afectada por factores adicionales, como la granulometría, la uniformidad del tamaño de partícula y la clorinación.

Los análisis típicos aplicados a la harina de trigos suaves incluyen: humedad, proteínas, cenizas, $\mathrm{pH}$, granulometría, almidón dañado, viscosidad, color, falling number, capacidad de retención de agua alcalina (AWR), farinógrafo o mixógrafo, extensógrafo o alveógrafo, factor de esparcimiento y prueba de horneo. Estos ensayos se realizan de acuerdo con los métodos establecidos por la American Asociation of Cereal Chemists (AACC, 1995). 
Conviene tener en cuenta que cuando se utilizan equipos como el farinógrafo, el mixógrafo, el extensógrafo o el alveógrafo para evaluar la harina de trigos suaves, no debe asumirse que las propiedades reológicas de la harina de trigo suave son las opuestas a las de la harina de trigos duros. Dicho de otra forma, si una buena harina de trigo duro da una curva fuerte, luego una harina de trigo suave debería dar una curva débil. Las propiedades fisicoquímicas de una buena harina de trigo suave son una combinación única de sus propiedades más que las propiedades de una harina de trigo dura de mala calidad.

\section{CLORINACIÓN}

La clorinación es el proceso por el cual la harina es tratada con gas cloro a un nivel de 0,5 a 2,5 onzas ( 14,17 a 70,87 gramos) por cada 100 libras (45,36 kilos) de harina, con el fin de reducir el $\mathrm{pH}$ de la harina y mejorar su calidad; se aplica solo a las harinas de trigo suave destinadas a la producción de bizcochos. Generalmente le sigue un tratamiento con peróxido de benzoílo, para remover cualquier residuo de color después de la clorinación. Este blanqueo adicional no altera la funcionalidad de la harina sino que únicamente le da a esta un color blanco.

La clorinación reduce el $\mathrm{pH}$ de la harina de manera que el $\mathrm{pH}$ resultante proporciona un índice parcial del nivel de clorinación obtenido. Sin embargo, el contenido de cenizas de la harina también determina la cantidad de cloro requerido para obtener resultados satisfactorios. El mejor comportamiento de la harina en la producción de bizcochos se obtiene cuando la harina ha sido correctamente blanqueada a un $\mathrm{pH}$ entre 4,6 y 5,1 . El pH de la harina sin blanquear está normalmente entre 5,8 a 6,1 .

La acción del cloro en la harina es un proceso complejo no del todo entendido. Altera las propiedades funcionales de los componentes de la harina, como los lípidos, las proteínas y las sustancias solubles en agua mientras estas modificaciones sean mantenidas a un nivel bajo.

La clorinación de la harina de trigo para la producción de bizcochos fue reportada por primera vez por Montzheimer (1931) y sus efectos fueron confirmados por Smith (1932). Ambos investigadores observaron que la miga de los bizcochos hechos con harina clorinada presentaba una textura más suave y pareja que la de los bizcochos hechos 
con harina sin clorinar o harina tratada con cualquier otro agente blanqueador. Smith también observó un mayor volumen y mejor simetría en los bizcochos hechos con harina clorinada.

Muchos investigadores han estudiado el modo de acción del cloro en la harina. Cuando la harina clorinada es fraccionada y analizada para estudiar la distribución del cloro, la mayor parte de este es encontrado en la proteína. Sollars (1960) descubrió que la mayor parte del cloro estaba distribuido en el gluten y en las fracciones de proteína solubles en agua. Chamberlain (1962) clasificó, por medio de aire, harina clorinada y sin clorinar en fracciones con elevado y reducido contenido de proteínas, respectivamente. El análisis de estas fracciones mostró que la tercera parte del cloro fue captado por los lípidos de la harina, la mitad por la proteína y de un quinto a un séptimo por los carbohidratos. Wilson et al. (1964) concluyeron que la distribución de cloro estaba relacionado con el área superficial de las partículas; consecuentemente, las fracciones más finas con elevado contenido de proteína enlazan más al cloro. Sollars (1961) utilizó el fraccionamiento de harina clorinada con agua y ácido acético para determinar la distribución de cloro. Encontró la mitad del cloro en la porción soluble en agua, lo cual le sugirió que el cloro había sido removido durante la etapa de separación. La mayor parte del gluten con contenido de cloro fue extraído con butanol. Algunas porciones de este lípido extraíble con butanol pudieron haber sido extraíbles con hexano antes de la extracción con agua y ácido acético (Olcott y Mecham, 1947; Davies et al., 1969). Cuando se consideran las diferencias entre lípidos libres y ligados, Sollars y Chamberlain coinciden. Gilles et al. (1964) reportaron que el $90 \%$ del cloro se recuperaba en las fracciones solubles en lípidos y agua y que prácticamente nada se encontraba en la fracción de almidón. Ewart (1968) observó que la reacción del cloro con la cisteína y metionina produjo ácido cisteico y sulfóxido de metionina. También observó la destrucción de la tirosina y de la histidina.

De estos trabajos se concluyó que la mayor parte de la reacción ocurría con las fracciones de proteína y lípidos. Sin embargo, Lamb y Bode (1963) fraccionaron húmedamente la harina, clorinaron las fracciones y las recombinaron con fracciones sin clorinar. Cuando la harina recombinada fue utilizada en la producción de bizcochos, los resultados demostraron que la clorinación del almidón fue la principal responsable de la mejora en la calidad de la harina. Sollars (1958a) también había reportado que tanto la fracción del gluten como la del 
almidón estaban relacionadas con el aumento en la calidad de la harina. Los cambios críticos en la calidad de esta harina podrían no estar reflejados en la distribución cuantitativa del cloro antes de reaccionar.

Son muchos los investigadores que han mostrado claramente que el primer efecto del cloro se produce sobre el almidón y que esta reacción mejora la calidad de la harina para la elaboración de bizcochos (Sollars, 1958a; 1958b; Lamb y Bode, 1963; Sollars y Rubenthaler, 1971; Johnson y Hoseney, 1979). Sin embargo, la siguiente pregunta subsiste: ¿cómo es afectado el almidón?

Kulp et al. (1972) reportaron estudios en los cuales la harina comercial de trigo suave fue tratada con cloro a niveles de 0 a 16 onzas (0 a 453,5 gramos) por 100 libras (45,36 kilos) de harina. Ellos observaron que el tratamiento redujo el $\mathrm{pH}$ y blanqueó los pigmentos de la harina, pero la mejora de sus características para la producción de bizcochos fue obtenida solo por debajo de las 4 onzas (113,4 gramos) por 100 libras de harina. Los investigadores mencionados atribuyeron estos cambios en las propiedades funcionales de la harina a la ruptura de los enlaces de hidrógeno inter e intramoleculares de las proteínas al igual que por la partición de los enlaces peptídicos, reacción que causa un aumento en la dispersibilidad y solubilización del gluten.

Kissel et al. (1979) demostraron que los lípidos presentes en la harina mejoran funcionalmente mediante el tratamiento de la harina con cloro hasta 2 onzas (56,7 gramos) por 100 libras (45,36 kilos) de harina. Por su parte, Johnson et al. (1979) concluyeron, basados en resultados obtenidos en estudios relacionados con la extracción de lípidos con éter en harinas clorinadas y sin clorinar, que los efectos beneficiosos producidos por la clorinación parecen estar en uno o varios componentes de la harina pero no en los lípidos. En una extensión de este estudio, Johnson y Hoseney (1979) encontraron que la clorinación de la harina tenía un efecto favorable en el volumen del bizcocho, el cual tendía a disminuir para el caso de harinas sin clorinar, mientras que el contenido de manteca aumentaba en la fórmula del bizcocho. Los estudios de fraccionamiento y reconstitución de harinas clorinadas y sin clorinar llevó a estos investigadores a concluir que la fracción de almidón era la responsable de las mejoras en la calidad de la harina clorinada.

Huang et al. (1982), utilizando procedimientos de clasificación con aire para fraccionar harinas para bizcochos tratadas con 0,1 y 4 onzas 
(0, 28,35 y 113,40 gramos) de cloro por 100 libras (45,36 kilos) de harina encontraron que la proteína absorbía significativamente más cloro que el almidón a todos los niveles de clorinación, y que la absorción de cloro por parte de la proteína aumentaba a niveles más elevados de clorinación mientras que la absorción de cloro por parte del almidón alcanzaba una constante a una dosis de 2 onzas (56,7 gramos) por 100 libras (45,36 kilos) de harina. Se obtuvo evidencias que indicaron una despolimerización y oxidación del almidón durante la clorinación. Se determinó que la capacidad de absorción y solubilidad de los gránulos de almidón a $149^{\circ} \mathrm{C}\left(90^{\circ} \mathrm{C}\right)$ fue mayor en masas de bizcochos hechas con harina clorinada en comparación con las hechas utilizando harina sin clorinar. Esto coincide con un nivel más elevado de actividad del agua a $176^{\circ} \mathrm{C}\left(80^{\circ} \mathrm{C}\right)$ observadas en masas hechas con harinas clorinadas.

De acuerdo con Hoseney (1994), la harina para bizcochos de ratio elevado (aquellos que contienen más azúcar que harina) debe ser tratada con gas cloro, el cual reacciona con prácticamente todos los componentes de la harina. El ritmo de reacción es rápido con la harina y lenta con el almidón. Sin embargo, estudios de fraccionamiento y reconstitución han demostrado que la reacción con el almidón es la responsable de la mejora en las propiedades de la harina. La cantidad de cloro es crítica. Cuando el cloro reacciona con materiales orgánicos, generalmente se produce ácido clorhídrico. Consecuentemente, el $\mathrm{pH}$ de la harina es una buena indicación del nivel de reacción y por ende de la cantidad de cloro añadido. Generalmente se desea un rango de $\mathrm{pH}$ de 4,7-4,9 para bizcochos de ratio elevado. La importancia actual del efecto del cloro parece estar en su reacción con el almidón para dar un almidón modificado (oxidado), que puede dilatarse más que el normal. Esto produce una masa (batido) más viscosa, a la misma temperatura, que una masa (batido) de una harina sin oxidar. La mayor viscosidad de la masa (batido) previene que el bizcocho colapse durante el horneado y, de alguna manera, después de ser retirado del horno.

\section{GRANULOMETRÍA}

Siempre ha sido considerada importante una granulometría fina y uniforme en el comportamiento de la harina para la producción de bizcochos. En general, cuanto más fina y uniforme sea la harina tiende a facilitar la clorinación a niveles bajos y con buenos resultados al 
momento del horneado de la masa de bizcocho. Normalmente, la harina para bizcochos se reduce en el molino hasta lograr que pase por una malla de seda 14XX. La relación actual entre la granulometría de la harina y sus propiedades dentro de la producción de bizcochos no está completamente establecida, aparte de la idea general de que la granulometría fina y uniforme promueve la dispersión de la harina en la masa de bizcocho. Estudios de molienda con pines y clasificación con aire de harinas para bizcochos han demostrado que algunas harinas son mejoradas mediante la remoción de las partículas más finas, dado que estas tienen mayor cantidad de proteínas.

La clasificación por aire ha sido quizás la manera clásica de demostrar la influencia del tamaño de las partículas de la harina en la calidad del bizcocho. Wischer (1958) demostró que la harina con elevado contenido de almidón, bajo contenido de proteínas y tamaño de partícula intermedio era la más adecuada para la producción de bizcochos. Exámenes microscópicos de estas fracciones mostraron que contenía gránulos de almidón grandes completamente separados de la proteína. Miller et al. (1967) observaron que la molienda con pines mejoraba la calidad de la harina para bizcochos pero la molienda excesiva la reducía. Yamasaki y Donelson $(1972,1973)$ mostraron, con muchas harinas, que la calidad de estas luego de la clorinación se correlacionaba con los tamaños pequeños o diámetro promedio de las partículas de harina. Esto sugiere que la mayor área superficial de las partículas pequeñas de la harina permite una clorinación más eficiente.

\section{CONCLUSIONES}

La clorinación de la harina de trigo suave fue introducida a principios de 1930 y es necesaria para producir bizcochos de ratio elevado con características óptimas de calidad. Es generalmente un proceso continuo en el cual se inyecta gas a pasajes de harina recién molida. El rango normal de clorinación es 1.100 a 2.300 ppm. A pesar de que el mecanismo exacto aún es incierto, los bizcochos producidos con harina clorinada óptimamente y a niveles óptimos de absorción tienen mejor volumen, grano más uniforme, color de miga más blanca (Sollars, 1958), mejor simetría (Gough et al., 1978) y mejores características sensoriales (Gaines y Donelson, 1982). El gas cloro reacciona rápidamente con la harina en una reacción dependiente de la 
superficie (Kesell y Yamasaki 1979). Esencialmente todos los componentes de la harina (gluten, almidón, lípidos, compuestos solubles en agua y pentosas) son modificados químicamente durante la reacción (Stauffer, 1990). La utilización del gas cloro en un molino conlleva problemas de seguridad. Adicionalmente, el público cuestiona la utilización de ciertos productos químicos en el procesamiento de alimentos, que determina la necesidad de encontrar un reemplazo adecuado para la clorinación de harinas de trigo.

Russo y Doe (1970) reportaron que el tratamiento térmico de la harina de trigo (sin clorinar) mejoraba sus propiedades para la producción de bizcochos. Ellos encontraron que la temperatura óptima de tratamiento era $120^{\circ} \mathrm{C}$, pero concluyeron que el tiempo de tratamiento no era importante. El comportamiento de la harina fue mejorado cuando el contendido de humedad de la harina fue reducida a menos de 7\%. A pesar de que las características de la harina fueron mejoradas en comparación con los de la harina sin clorinar; la calidad de los bizcochos no fue igual a la de aquellos hechos con harina clorinada. Los autores notaron que fueron necesarios cambios en la formulación para producir bizcochos satisfactorios. Una patente reciente (Rabe y Meyers, 1995) detalla algunos cambios beneficiosos en la formulación.

Johnson y Hoseney (1979) estudiaron el efecto del calentamiento y almacenamiento de harinas. La harina almacenada a temperatura ambiente durante dos meses resultó en bizcochos con mayor volumen y grano. Estos cambios relacionados con el "envejecimiento" fueron acelerados mediante temperatura. El tratamiento de harinas frescas (recién molidas) mejoró el grano y eliminó el colapsamiento durante el horneo.

Thomasson et al. (1995) reportaron el tratamiento de harinas de trigo suave (sin clorinar) a $7 \%$ de humedad por 30 minutos a $125^{\circ} \mathrm{C}$; suplementada con $0,12 \%$ (basado en el peso de la harina) de goma xantan produjo bizcochos con volúmenes que fueron ligeramente mayores que aquellos bizcochos hechos con harina clorinada. Las características del grano fueron esencialmente equivalentes. La adición de 200 a 300 ppm de L-cisteína a la masa del bizcocho hecho con harina sin clorinar tratada térmicamente también produjo bizcochos con características de volumen y grano similares a los bizcochos hechos con harina clorinada. El tratamiento térmico también redujo el grado de encogimiento del bizcocho durante el horneado a un valor equivalente a 
aquellos bizcochos hechos con harina clorinada. Los valores de entalpía de harinas tratadas térmicamente con calorimetría diferencial mostraron que el almidón no fue gelatinizado durante el calentamiento.

\section{BIBLIOGRAFÍA}

American Association of cereal Chemists. Approved methods of the American Association of Cereal Chemists. St. Paul, MN, 1995.

Chamberlain, N. "What goes into a Chorleywood? How confectionery fits into research program". Bakers Rev. 79:2014, 2016, 2033, 1962.

Davies, R. J.; Daniels, N. W. R. y R. N. Greenshields. "An improved method of adjusting flour moisture in studies on lipid binding". J. Food Technol. 4:117-123, 1969.

Ewart, J. A. D. "Action of glutaraldehyde, nitrous acid or chlorine on wheat proteins". J. Sci. Food Agric. 19:370-373, 1968.

Gaines, C. S. y J. R. Donelson. "Cake batter viscosity and expansion upon heating". Cereal Chem. 59:237-240, 1982.

Gilles, K. A.; Kaelble, E. F. y V. L. Youngs. "X-ray spectrographic analysis of chlorine in bleached flour and its fraction". Cereal Chem. 41:412-424, 1964.

Goughb B. M.; Whitehouse, M. E. y C. T. Greenwood. "The role and function of chlorine in the preparation of high ratio cake flour". Crit. Rev. Food. Sci. and Nutr. 10:91-110, 1978.

Grau, H.: Wehrle, K., y E. K. Arendt. "Evaluation of a two step baking procedure for convenience sponge cakes". Cereal Chem. 76(2): 303-307, 1999.

Hoseney, R. C. Principles of cereal science and Technology. 2. ${ }^{\text {a }}$ edición. Saint Paul, Minnesota: American Association of Cereal Chemists, 1994.

Huang, G.; Finn, J. W. y E. Varriano-Marston. "Flour chlorination. II. Effects of water binding". Cereal Chem. 59:500-506, 1982.

Johnson, A. C.; Hoseney, R. C. y E. Varriano-Marston. "Chlorine treatment of cake flours". Cereal Chem. 56:333, 1979. 
Johnson, A. C. y R. C. Hoseney. "Chlorine treatment of cake flours. III. Fractionation and reconstitution techniques for $\mathrm{Cl}_{2}$-treated and untreated flours". Cereal Chem. 56:443-445, 1979.

_ "Chlorine treatment of cake flours. II. Effect of certain ingredients in the cake formula". Cereal Chem. 56:336-338, 1979.

Kissel, L. T. y W. T. Yamasaki. "Cake-baking dynamics: Relation of flour chlorination rate to batter expansion and layer volume". Cereal Chem. 56:324-327, 1979.

Kissel, L. T.; Donelson, J. R., y R. L. Clements. "Functionality in white layer cake of lipids from untreated and chlorinated patent flours, I. Effects of free lipids". Cereal Chem. 56:11-14, 1979.

Kulp, K.; Tsen, C. C. y C. J. Daly. "Effect of chlorine on starch component of soft wheat flour". Cereal Chem. 49:194-200, 1972.

Lamb, C. A. y C. E. Bode. "Quality evaluation of soft wheat". Ohio Agric. Exp. Stn. Res. Bull. 926, 1963.

Miller, B. S.; Trimbo, H. B. y K. R. Powell. "Effects of flour granulation and starch damage on cake the making quality of soft wheat flour". Cereal Sci. Today 12:245, 250-257, 1967.

Montzheimer, J. W. "A study of methods for testing cake flour". Cereal Chem. 8:510-517, 1931.

Olcott, H. S. y D. K. Mecham. "Characterization of wheat gluten. I. Protein lipid complex formation during doughing of flours. Lipoprotein nature of glutenin fraction". Cereal Chem. 24:407-414, 1947.

Posner, E. y A. Hibbs. Wheat flour milling. St. Paul, Minnesota: American Association of Cereal Chemists, Inc., 1997.

Pyler, E. J. Baking science and technology. Merriam, Kansas: Sosland Publishing Company, 1988.

Rabe, G. O. y T. A. Meyers. "High ratio baking composition. Patent W095/057431995”, 1995.

Russop, J. V. B. y G. A. F. Doe. "Heat-treatment of flours as an alternative to chlorination". J. Food Techol. 5:363-374, 1970.

Smith, E. E. "Report of subcommittee on hydrogen-ion concentration with special reference to the effect of flour bleach". Cereal Chem. 
9:424-428, 1932.

Sollars, W. F. "Cake and cookie flour fractions affected by chlorine bleaching”. Cereal Chem. 35:100-110. 1958.

- "Fractionation and reconstitution procedures for cake flours". Cereal Chem. 35:85-99, 1958a.

—. "Cake and cookie flour fractions affected by chlorine bleaching". Cereal Chem. 35:100-110, 1958b.

- "Chloride content of cakes flours and flour fractions". Cereal Chem. 38:487-500, 1961.

Sollars, W. F. y G. L. Rubenthaler. "Performance of wheat and other starches reconstituted flours”. Cereal Chem. 48:397-410, 1971.

Stauffer, C. E. "Oxidants". Functional additives of bakery foods. Nueva York: Van Nostrand Reinhold, 1990.

Thomasson, C. A.; Miller, R. A. y R. C. Hoseney. "Replacement of chlorine treatment for cake flour". Cereal Chem. 72(6):612-620, 1995.

Yamasaki, W. T. y D. H. Donelson. "Evaluating soft wheat quality of early generation progenies". Crop Sci. 13:374-375, 1973.

- "The relationship between flour particle size and cake-volume potential among Eastern soft wheats". Cereal Chem. 49:649653,1972 .

Wichser, F. W. "Air-classified flour fractions". Cereal Sci. Today 3:123-126, 1958.

Wilson, J. T.; Donelson, D. H. y C. R. Sipes. "Mechanism of improver action in cake flours. I. The relation between flour specific surface and chlorine distribution". Cereal Chem. 30:242-246, 1964. 